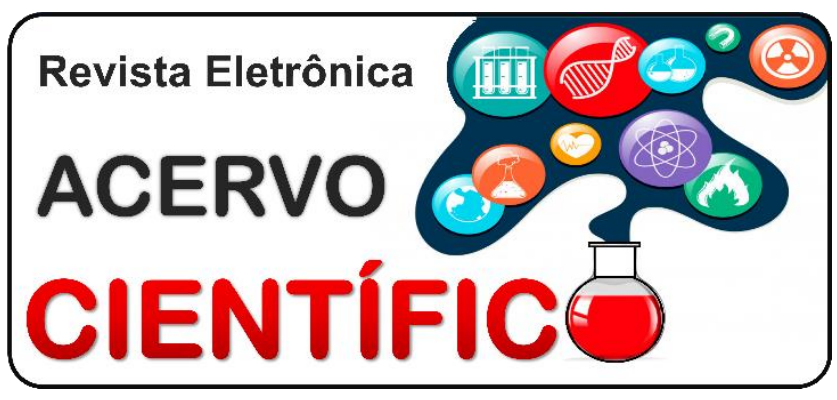

\section{REVISÃO BIBLIOGRÁFICA}

Recebido em: 12/2020

Aceito em: 1/2021

Publicado em: 2/2021

\title{
Métodos não invasivos de monitorização hemodinâmica em pacientes acometidos por choque circulatório: uma revisão de literatura
}

\author{
Non-invasive hemodynamic monitoring methods in patients affected by circulatory shock: \\ a literature review
}

\section{Métodos de monitorización hemodinámica no invasiva en pacientes afectados por shock circulatorio: revisión de la literatura}

Ana Kelly Teixeira Rocha ${ }^{1 *}$, Paulo Cézar Tostes de Campos Júnior ${ }^{1}$, Ayla Gerk Rangel ${ }^{2}$, Fernanda Gadelha Fernandes ${ }^{3}$, Karen Aragão Muniz Rodrigues ${ }^{4}$, Luanna Bastos de Souza Eller ${ }^{5}$, Maria Angélica Otero de Melo dos Reis ${ }^{6}$, Tamara Araújo Pereira Campos ${ }^{7}$, Thaiz Geovana Bezerra ${ }^{8}$, Vitória Ragiotto dos Santos ${ }^{9}$.

\begin{abstract}
Resumo: Esse artigo tem como propósito observar os tipos de monitorização hemodinâmica comparando seus benefícios específicos, limitações e aplicações em pacientes críticos e chocados, tendo como foco métodos não invasivos em pacientes com choque circulatório. $O$ trabalho se baseou em investigar literatura e estudos anteriores analisando os principais métodos não invasivos existentes destacando prós, contras e importância de cada um, tendo como propósito final uma contribuição para o monitoramento de pacientes acometidos por choque circulatório. Vale ressaltar também, que esse artigo abordou os métodos de monitorização não invasivos de forma a destacar as vantagens de cada método, uma vez que tais técnicas tem ganhado destaque na medicina em virtude dos avanços tecnológicos que permearam essa área. Tal temática tem papel de destaque no que tange cuidados aos pacientes, pois essas técnicas constituem componente essencial no atendimento eficaz do paciente criticamente doente já que o estado hemodinamicamente instável apresenta um prognóstico grave com alto risco de evolução para choque circulatório e óbito em um curto espaço de tempo, sendo indispensável sua monitorização.
\end{abstract}

Palavras-chave: Monitorização hemodinâmica, Choque circulatório, Cuidados críticos.

\footnotetext{
Abstract: This article aims to observe the different types of hemodynamic monitoring comparing their benefits, limitations and applications in critical patients and shock, having as focus the noninvasive methods in patients under circulatory shock. This work consisted in investigating preexistent literature and studies analyzing the most used non-invasive methods highlighting the benefits, prós, cons and importance of each one. Having as

${ }^{1}$ Centro Universitário de Caratinga (UNEC), Caratinga - MG. *E-mail: anakelly.01@hotmail.com

2Universidad Católica Argentina (UCA), Buenos Aires - AR.

UUniversidade Potiguar (UNP), Natal - RN.

${ }^{4}$ Universidade Federal de Campina Grande (UFCG), Cajazeiras - PB.

5Universidade Vila Velha (UVV), Vila Velha - ES.

GUniversidad Nacional do Rosário (UNR), Rosário - AR.

${ }^{7}$ Faculdade da Saúde e Ecologia Humana (FASEH), Vespasiano - MG.

8Universidade do Oeste Paulista (UNOESTE), Jaú - SP.

${ }^{9}$ São Leopoldo Mandic (SLM), Campinas - SP.
} 
a final purpose a contribution to the hemodynamic monitoring of circulatory shock patients. It is also worth mentioning that this article addressed non-invasive monitoring methods in order to highlight the advantages of each method, since such techniques have gained prominence in medicine due to the technological advances that have permeated this area. Such theme has a prominent role when it comes to patient care, as these techniques are an essential component in the effective care of critically ill patients since the hemodynamically unstable state presents a severe prognosis with a high risk of evolution to circulatory shock and death in a short space of time, monitoring is essential.

Key words: Hemodynamic monitoring, Circulatory shock, Critical care.

Resumen: Este artículo tiene como objetivo observar los distintos tipos de monitorización hemodinámica comparando sus beneficios, limitaciones y aplicaciones en pacientes críticos y en shock, teniendo como enfoque métodos no invasivos en paciente con shock circulatorio. El trabajo consiste en investigar artículos y estudios ya existentes analizando los principales métodos no invasivos destacando los pros, contras y la importancia de cada uno, teniendo como propósito final una contribución para la monitorización hemodinámica de pacientes acometidos por shock circulatorio. También cabe mencionar que este artículo abordó los métodos de monitorización no invasivos con el fin de resaltar las ventajas de cada método, ya que dichas técnicas han ganado protagonismo en la medicina debido a los avances tecnológicos que han permeado esta área. Tal tema tiene un papel destacado en la atención al paciente, ya que estas técnicas son un componente esencial en la atención eficaz del paciente crítico, ya que el estado hemodinámicamente inestable presenta un pronóstico severo con alto riesgo de evolución a shock circulatorio y muerte en una breve espacio de tiempo, el seguimiento es fundamental.

Palabras clave: Monitorización hemodinámica, Shock circulatorio, Cuidados críticos.

\section{INTRODUÇÃO}

A monitorização hemodinâmica (MHD) é um componente essencial no atendimento eficaz do paciente criticamente doente. $O$ espectro vai desde a avaliação clínica de rotina até procedimentos mais complexos realizados de forma invasiva e não invasiva. A abordagem não invasiva vem ganhando destaque e devido a sua constante evolução tecnológica e técnica está se tornando um importante meio de avaliação de pacientes em choque circulatório ou com risco (LAHER AE, et al., 2017). As principais formas não invasivas para a compreensão da instabilidade hemodinâmica é realizada por meio de ecocardiograma, ultrassonografia, Doppler Esofágico, Bioreactância, dispositivos de monitoramento de dedo como o Oxímetro e ClearSight, entre outras (LAHER AE, et al., 2017; HAMZAOUI O, et al., 2015; JOZWIAK M, et al., 2015; GUARRACINO F, et al., 2018).

O estado hemodinamicamente instável apresenta um prognóstico grave com alto risco de evolução para choque circulatório e óbito em um curto espaço de tempo, cuja expressão clínica está relacionada a ineficiente perfusão tissular (GASPAR A, et al., 2018). O choque circulatório tem origens diversas, e pode apresentar variáveis e perfis hemodinâmicos distintos, logo deve-se priorizar métodos e técnicas de diagnóstico eficazes para possibilitar uma conduta terapêutica adequada para cada etiologia (GASPAR A, et al., 2018; JOZWIAK M, et al., 2015).

O diagnóstico preciso de choque circulatório em pacientes críticos assistidos na unidade de terapia intensiva (UTI) é algo dificultoso, principalmente pelo fato de que, apenas com a avaliação clínica, não é possível em alguns casos. A MHD se faz essencial para evitar a prevenção e agravos das diferentes condições cardiovasculares que causam os choques circulatórios em tais pacientes, e garante uma maior chance de tratamento imediato e adequado para garantir excelência terapêutica (HAMZAOUI O, et al., 2015; JOZWIAK M, et al., 2015).

O presente estudo busca revisar a literatura científica a respeito das técnicas de monitoramento hemodinâmico, reforçando a tendência e importância da utilização das técnicas não invasivas e sua evolução 
contínua, como ferramenta essencial de diagnóstico em UTIs, ressaltando o fato de que estes são métodos efetivos, seguros e eficazes em termos de custos, além de gerarem menos complicações em relação aos métodos invasivos.

\section{REVISÃO BIBLIOGRÁFICA}

\section{Choque circulatório}

O choque circulatório se define pela má perfusão do sangue devido a distintas ocorrências que levam a incapacidade de manter o suprimento de sangue necessário nos tecidos, o qual pode levar à falência circulatória gerando um estado de hipoperfusão e hipotensão. A hipóxia celular prolongada, resultante da má perfusão, leva a morte tecidual e posterior falência de órgãos culminando em situações extremas de morte de paciente críticos, em caso não detectados antecipadamente (GASPAR A, et al., 2018). Devido a estes fatores, a coleta de dados fisiológicos de pacientes criticamente enfermos se faz imprescindível (TERAN F, 2019).

As alterações hemodinâmicas se apresentam em consequência de fatores como a hipovolemia, obstruções do fluxo sanguíneo, embolia pulmonar ou pneumotórax, falência da função de bombeamento cardíaco e distribuição inadequada do fluxo sanguíneo corporal decorrente de alterações no tônus vascular (RUSSEL A, et al., 2020; CECCONI M, et al., 2014). Manifestando parâmetros hemodinâmicos de pré-carga, pós-carga e contratilidade que determinam o perfil hemodinâmico da sua condição de origem, além de sua apresentação clínica (AZCARATE JMA, et al., 2012).

\section{Tipos de Choque}

O choque circulatório pode ser classificado como: cardiogênico, hipovolêmico, obstrutivo e distributivo, onde os três primeiros estão associados a um estado de fluxo sanguíneo reduzido, e o último a um estado hipercinético (GUARRACINO F, et al., 2018; RUSSELL A, et al., 2020; KOSTER G e VAN DER HORST ICC, 2017; LAHER AE, et al., 2017; CECCONI M, et al., 2014).

Cada tipo de choque possui manifestações específicas, em casos de redução do Débito Cardíaco (DC) em consequência da diminuição do volume sanguíneo circulante configura-se o chamado choque hipovolêmico. Já em situações onde ocorre a obstrução de fluxo circulatório se denomina choque obstrutivo. $\mathrm{Na}$ ocorrência de alterações na função cardíaca ou a variações no fluxo sanguíneo caracteriza-se o choque cardiogênico. E finalmente, em casos de alteração da distribuição de fluxo sanguíneo que resulta em falhas de perfusão, classifica-se como choque distributivo. Estas manifestações têm potencial sobreposição e pacientes com apenas um tipo de choque também podem manifestar as outras formas simultaneamente (CECCONI M, et al., 2014; KOSTER G e VAN DER HORST ICC 2017).

\section{Manifestações Clínicas}

Clinicamente, o choque circulatório se manifesta, em geral, por hipotensão, que nem sempre estará presente devido a ação dos mecanismos fisiológicos de compensação, e sinais de prejuízo da microcirculação com redução da perfusão periférica, que se expressam nas extremidades corporais com baixas temperaturas e cianose, manchas cutâneas, redução da velocidade de enchimento capilar, débito urinário inferior a 0,5 $\mathrm{mL} / \mathrm{kg} /$ hora e alteração do estado mental, com confusão e desorientação (GASPAR A, et al., 2018; CECCONI M, et al., 2014).

Algumas das consequências da isquemia tecidual decorrente do choque circulatório, causadas pela deficiência na distribuição e utilização de oxigênio pelas células corporais, são: lesão tecidual; disfunção e morte celular e falência de múltiplos órgãos, e possível óbito. (RUSSEL A, et al., 2020; LAHER AE, et al., 2017; GASPAR A, et al., 2018; CECCONI M, et al., 2014). As complicações secundárias do choque em sua maioria são tempo-dependentes assim, o manejo precoce da MHD para evitar complicações é essencial (LAHER AE, et al., 2017).

O diagnóstico do choque se baseia na combinação de sinais clínicos e alterações hemodinâmicas e bioquímicas. Logo, a escolha de uma técnica apropriada e eficaz de MHD deve levar em consideração a fase 
do choque, a complexidade do estado hemodinâmico e a resposta à terapia inicial aplicada (KOSTER G e VAN DER HORST ICC 2017; JOZWIAK M, et al., 2018).

\section{Métodos não invasivos de monitorização hemodinâmica}

Existem inúmeros métodos de MHD, porém na atualidade destacam-se as técnicas não invasivas e sua evolução, buscando sempre o melhor prognóstico para os pacientes.

\section{Doppler Esofágico}

É um exame de imagem fundamentado na verificação da velocidade do fluxo de sangue da artéria aorta descendente através de um transdutor situado no limite distal de uma sonda maleável. Este dispositivo é inserido por via oral, atingindo o nível médio do tórax (ROCHA PN, et al.,2010). Tal equipamento é capaz de mensurar constantemente o fluxo de sangue da artéria aorta ascendente, o diâmetro da aorta, e a frequência cardíaca (JOZWIAK M, et al., 2015). Além disso, oferece instrumentos hemodinâmicos como a aceleração média e o pico de velocidade do fluxo sistólico do sangue aórtico, que podem detectar alterações na pressão sistólica (JOSWIAK M, et al., 2018). A precisão desta modalidade de exame sofre influência do movimento da sonda no esôfago, portanto recomenda-se que seja realizado em bloco cirúrgico para obtenção de resultados fidedignos (JOZWIAK M, et al., 2018).

\section{Ultrassom}

É um exame de imagem seguro, isento de radiação ionizante e que pode ser feito em beira leito. Nota-se uma progressão da sua utilização em UTIs e departamentos de emergência (LAHER AE, et al., 2017). Este exame possibilita a avaliação do sistema cardiovascular, identificando diversos distúrbios de etiologia circulatória, como redução da fração de ejeção e dilatação das câmaras cardíacas, além de evidenciar sinais de emergência como choque circulatório e tamponamento cardíaco (RUSSEL A, et al., 2020). A avaliação clínica associada à ultrassonografia deve ser a primeira conduta para avaliação do estado hemodinâmico de um paciente em choque circulatório, permitindo a escolha da intervenção terapêutica inicial e de outros métodos de monitorização adequados (LAHER AE, et al., 2017).

\section{Ecocardiografia Transtorácica com estudo Doppler (eco-Doppler)}

Essa técnica também pode ser realizada à beira leito e permite avaliação cardíaca morfológica e funcional. Recentemente vem sendo o método de escolha para a MHD e avaliação primária do paciente em choque circulatório, cada vez mais presente nas UTIs (RUSSEL A, et al., 2020; GASPAR A, et al., 2018; CECCONI $M$, et al., 2014). Este é fundamentado na detecção, através de um transdutor, da velocidade do fluxo sanguíneo pela emissão de ondas de ultrassom ao transpassar os vasos sanguíneos (RUSSEL A, et al., 2020; CECCONI M, et al., 2014).

O transdutor de ultrassom verifica o volume sistólico e DC por meio da identificação do fluxo de sangue proveniente da valva aórtica e pulmonar. O exame eco-doppler transtorácico simplificado, usado para verificação do fluxo sanguíneo nas valvas semilunares, torna-se literalmente não-invasivo ao ser associado à manobra EPP (ROCHA PN, et al., 2010). Entretanto, não permite análise contínua dos padrões cardiocirculatórios, sendo essa uma característica desfavorável em relação aos métodos invasivos. (CECCONI M, et al., 2014).

Profusos parâmetros podem ser avaliados e ponderados, em particular o DC, a pressão venosa central, as resistências vasculares periféricas e as pressões de enchimento do ventrículo esquerdo (GASPAR A, et al., 2018). Esse exame, associado a achados clínicos e à monitorização básica permite entender a causa da instabilidade hemodinâmica, sendo empregado como método de diagnóstico, e eleito a terapia de suporte mais adequada ao paciente, além de avaliar a resposta do paciente às intervenções terapêuticas (GASPAR A, et al., 2018; SAUGEL B e VINCENT JL, 2018; CECCONI M, et al., 2014). E, tendo em vista que não há relação linear entre o estado hemodinâmico dos pacientes e a função sistólica dos ventrículos cardíacos, a avaliação do DC por meio deste, se dá de forma semiquantitativa como muito baixo, baixo, normal ou elevado. É um dos principais parâmetros de escolha para o MHD dos pacientes em estado grave, tendo fundamental importância a sua monitorização e otimização para a estabilidade circulatória, especialmente em pacientes 
com choque circulatório refratário ou persistente após conduta terapêutica inicial de ressuscitação (GASPAR A, et al., 2018; SAUGEL B e VINCENT JL, 2018). Contudo, apesar de suas significativas vantagens, não apresenta indicação para substituição dos métodos semi-invasivos e invasivos de MHD contínua, mas sim como método complementar e de eleição na avaliação preliminar dos pacientes em falência circulatória (GASPAR A, et al., 2018).

\section{Cardiografia por bioimpedância transtorácica (ICG)}

Essa técnica utiliza mudanças cíclicas na impedância elétrica torácica induzidas pelos batimentos cardíacos para estimar mudanças no volume sanguíneo no interior da aorta e mudanças no volume de fluido do tórax, os quais fornecem informações sobre o status volêmico do paciente (HAMZAOUI O, et al., 2015). Dessa forma, é possível estimar parâmetros hemodinâmicos e detectar precocemente o risco de descompensações. (HAMZAOUI O, et al., 2015; RUSSEL A, et al., 2020; CECCONI M, et al., 2014). Este permite a análise direta ou indireta dos quatro determinantes do DC, assim como os efeitos das medidas terapêuticas sobre cada um deles, auxiliando na escolha da classe e titulação das doses dos medicamentos (CECCONI M, et al., 2014; HAMZAOUI O, et al., 2015; RUSSEL A, et al., 2020).

A técnica é baseada na Lei de Ohm e são utilizados quatro pares de eletrodos posicionados no pescoço e no tórax do paciente, que são conectados a um aparelho portátil, de forma que os quatro sensores externos geram uma corrente constante de baixa frequência e alta amplitude e os eletrodos internos captam as mudanças instantâneas de voltagem (HAMZAOUI O, et al., 2015). As variações em relação à impedância basal ocorrem devido a modificações no volume pulmonar com a respiração e mudanças no volume sanguíneo no interior de grandes vasos durante o ciclo cardíaco. Os parâmetros hemodinâmicos resultantes da ICG podem auxiliar no diagnóstico, no prognóstico e na orientação da terapêutica (CECCONI M, et al., 2014; HAMZAOUI O, et al., 2015; RUSSEL A, et al., 2020). As principais limitações do método são o aumento do volume de fluido torácico, pacientes em ventilação mecânica invasiva, arritmias cardíacas e obesidade (HAMZAOUI O, et al., 2015).

\section{Ultrassom Point of Care (USPOC)}

É um método amplamente disponível e eficaz para avaliação hemodinâmica à beira leito em pacientes graves e internados em UTIs. Sua avaliação se estende principalmente ao coração, grandes vasos, pulmões e cavidades pleurais e abdômen (KOSTER G e VAN DER HORST ICC., 2017). O USPOC permite a determinação do tipo de choque, a avaliação da resposta ao tratamento e o acompanhamento da evolução clínica através de parâmetros como a colapsibilidade da veia cava inferior e a variação dos diâmetros e volumes das câmaras cardíacas e dos grandes vasos durante o ciclo cardíaco (KOSTER G e VAN DER HORST ICC., 2017; RUSSEL A, et al., 2020). Com isso, é possível a escolha da terapêutica mais eficaz, evitar intervenções potencialmente prejudiciais e estabelecer prognóstico (CECCONI M, et al., 2014; KOSTER G e VAN DER HORST ICC, 2017; RUSSEL A, et al., 2020) A técnica exige treinamento e conhecimento das técnicas de execução e interpretação dos achados pelo clínico (KOSTER G e VAN DER HORST ICC, 2017; RUSSEL A, et al., 2020).

\section{Biorreactância}

É um exame realizado por meio do posicionamento de eletrodos na superfície do tórax e do pescoço do paciente que mensuram o volume sistólico pela detecção de alterações na frequência da corrente elétrica circulante pelo tórax, em decorrência das variações do fluxo sanguíneo intratorácico oriundas de cada ciclo cardíaco. (RUSSEL A, et al., 2020; LAHER AE, et al., 2017; CECCONI M, et al., 2014; HAMZAOUI O, et al., 2015; JOZWIAK M, et al., 2018). Esse, permite um monitoramento contínuo do volume sistólico e do DC, tomando como base o retardo entre a corrente elétrica aplicada no tórax e a voltagem que retorna ao eletrodo. (LAHER AE, et al., 2017; CECCONI M, et al., 2014).

Uma das desvantagens é que a acurácia depende do posicionamento correto dos eletrodos, além de sua efetividade ser limitada em pacientes críticos e instáveis. (RUSSEL A, et al., 2020; HAMZAOUI O, et al., 2015; FAGNOUL D, et al., 2012; KUPERSZTYCH-HAGEGE E, et al., 2013). Outra desvantagem é a limitação de monitoramento dos parâmetros hemodinâmicos, sendo usado unicamente para avaliação do DC e volume 
sistólico (JOZWIAK M, et al., 2018). As evidências atuais para validação da eficácia da biorreactância como MHD são controversas, tornando seu uso restrito, mas estudos comprovam sua eficácia na avaliação do DC e fluido-responsividade quando associado à manobra de elevação passiva das pernas (LAHER AE, et al., 2017; BENOMAR B, et al., 2010).

\section{Ecocardiografia}

O ecocardiograma (ECO) pode ser usado na avaliação não invasiva (ecocardiograma transesofágico ETE) ou semi-invasiva (Ecocardiograma Tanstorácico (ETT)), da função e estrutura cardíaca, incluindo a fração de ejeção do ventrículo esquerdo, a função do ventrículo direito e medidas de status de volume, além disso, pode ser usada para estimar o volume sistólico e também o DC (OLIVIERI PP, et al., 2019; AZCARATE JMA, et al., 2011). A desvantagem se dá por ser operador-dependente. Além disso, são poucos os guidelines que orientem os parâmetros hemodinâmicos ecocardiográficos na UTI (MERCADAL JM, et al., 2017; AZCARATE JMA, et al., 2011). É o exame de primeira linha em pacientes que se encontram chocados, apesar de não ser uma técnica de monitoramento contínuo, sendo capaz de dizer quais os mecanismos envolvidos e a terapêutica mais adequada para cada caso (JOZWIAK M, et al., 2018). Os parâmetros hemodinâmicos mais importantes que podem ser obtidos em pacientes críticos são a avaliação da pré-carga, pós-carga e contratilidade. A partir dessas informações, podemos obter os perfis hemodinâmicos. Os objetivos são: Excluir doença cardíaca estrutural grave como causa de instabilidade hemodinâmica (ETE); Monitorar a função ventricular direita e esquerda; Monitorar parâmetros dinâmicos de pré-carga e contratilidade (AZCARATE JMA, et al., 2011).

\section{Exame Clínico}

Além de métodos tecnológicos e inovadores, o exame clínico também possui importância no contexto da análise hemodinâmica. Ele é composto por uma história clínica e um exame físico completo, associado a monitorização básica à beira do leito, permitindo entender a etiologia do choque circulatório e estabelecer uma escolha mais eficiente do método de monitorização adequado a cada paciente (LAHER AE, et al., 2017; GASPAR A, et al., 2018). Embora seja relativamente subjetivo, tem papel importante no diagnóstico de alterações hemodinâmicas dos pacientes em choque circulatório. No entanto, alguns achados clínicos podem ser oriundos de comorbidades associadas, a exemplo de débito urinário inadequado presente nas afecções renais e das alterações do nível de consciência em decorrência de fármacos ou drogas ilícitas, podendo interferir no diagnóstico de alterações hemodinâmicas em pacientes críticos (LAHER AE, et al., 2017).

\section{Exames laboratoriais com medição de biomarcadores do sistema circulatório}

A realização de exames laboratoriais com medição de biomarcadores do sistema circulatório, especialmente a saturação venosa de oxigênio (SvO2), e os níveis séricos de lactato, tem grande importância na monitorização de pacientes em falência circulatória (LAHER AE, et al., 2017; ROCHA PN, et al., 2010). Esses marcadores podem ser utilizados para identificar alterações como baixo DC e hipoxemia, que se apresentam com baixa ScvO2, e alterações no metabolismo e utilização do oxigênio, representadas pela hiperlactacidemia, sendo relevantes para avaliar a necessidade de intervenções terapêuticas específicas, como a administração de fluidos em pacientes com altos níveis de lactato (LAHER AE, et al., 2017; GASPAR A, et al., 2018; CECCONI M, et al., 2014). Altos níveis séricos de lactato são frequentemente associados com pior prognóstico em todos os tipos de choque, sendo úteis medidas seriadas dos valores sanguíneos de lactato para avaliação prognóstica e escolha terapêutica (CECCONI M, et al., 2014).

\section{CONSIDERAÇÕES FINAIS}

O presente artigo pretendeu estruturar uma série de considerações a respeito dos principais métodos de MHD não invasiva em pacientes acometidos por choque circulatório. Métodos não invasivos são efetivos, seguros, eficazes em termos de custo e geram menos complicações que métodos invasivos. Percebe-se que, na maioria dos casos, a avaliação clínica direcionada e a monitorização à beira-leito constituem manejo apropriado do paciente em choque. Para avaliação dos achados de instabilidade hemodinâmica, aplicam-se, com frequência, ultrassonografia, ecocardiograma, biorreactância, Doppler esofágico, dentre outros. A partir 
desses métodos, a medição contínua de determinados biomarcadores séricos, como a saturação venosa de oxigênio, o nível de lactato no sangue e o gradiente venoarterial de dióxido de carbono é vantajosa para a deteç̧ão de hipóxia e de perfusão tecidual. Ressalta-se, portanto, a importância do treinamento do emergencista para a apreciação desses marcadores e de como obtê-los, pois, são cruciais tanto para a profilaxia quanto para o prognóstico do choque e devem guiar a terapia apropriada para o paciente crítico. Conclui-se que o conhecimento abrangente dos métodos não invasivos e de quais são mais indicados para cada caso são essenciais para um desfecho positivo.

\section{REFERÊNCIAS}

1. RUSSELL A, et al. A Physiologic Approach to Hemodynamic Monitoring and Optimizing Oxygen Delivery in Shock Resuscitation. Journal of clinical medicine, 2020; 9(7): 2052.

2. LAHER AE, et al. A review of hemodynamic monitoring techniques, methods and devices or the emergency physician. American Journal of Emergency Medicine, 2017; 35: 1335-1347.

3. CECCONI M, et al. Consensus on circulatory shock and hemodynamic monitoring. Task force of the European Society of Intensive Care Medicine. Intensive Care Med, 2014; 40:1795-1815.

4. BENOMAR B, et al. Fluid responsiveness predicted by noninvasive bioreactance-based passive leg raise test. Intensive Care Med, 2010; 36:1875-81.

5. HAMZAOUI O, et al. Evolving concepts of hemodynamic monitoring for critically ill patients. Indian Journal of Critical Care Medicine, 2015; 19(4): 220-226.

6. FAGNOUL D, et al. Cardiac output measurements using the bioreactance technique in critically ill patients. Crit Care, 2012; 16: 460.

7. KUPERSZTYCH-HAGEGE E, et al. Bioreactance is not reliable for estimating cardiac output and the effects of passive leg raising in critically ill patients. Br J Anaesth, 2013; 111: 961-966.

8. JOZWIAK M, et al. Less or more hemodynamic monitoring in critically ill patients. Current Opinion in Critical Care, 2018; 24(4): 309-315.

9. JOZWIAK M, et al. Monitoring: from cardiac output monitoring to echocardiography. Current Opinion in Critical Care, 2015; $21: 395-401$.

10. GASPAR A, et al. Avaliação hemodinâmica não invasiva por ecocardiograma Doppler. Revista Brasileira de Terapia Intensiva, 2018; 30(3): 385-393.

11. ROCHA PN, et al. Avaliação hemodinâmica em paciente criticamente enfermo. Jornal Brasileiro de Nefrologia, 2010; 32(2): 201-212.

12. GUARRACINO F, et al. Novel applications of bedside monitoring to plumb patient hemodynamic state and response to therapy. Minerva Anestesiologica, 2018; 84(7): 858-864.

13. GREEN MS, et al. Near-Infrared Spectroscopy: The New Must Have Tool in the Intensive Care Unit? Seminars in Cardiothoracic and Vascular Anesthesia, 2016; 20: 213-224.

14. AZCARATE JMA, et al. Papel de la ecocardiografía en la monitorización hemodinámica de los pacientes críticos. Medicina Intensiva, 2012; 36(3): 220-232.

15. TERAN F. Resuscitative Cardiopulmonary Ultrasound and Transesophageal Echocardiography in the Emergency Department. Emergency Medicine Clinics of North America, 2019; 37(3): 409-430.

16. SAUGEL B e VICENT JL, et al. Consensus on circulatory shock and hemodynamic monitoring. Task force of the European Society of Intensive Care Medicine. Intensive Care Med, 2014; 40:1795-1815

17. LISSETH HGG, et al. Monitorización de la perfusión tisular en el paciente criticamente enfermo. Rev Cient Cienc Med, 2016; 19(2): 43-47.

18. GARCÍA JLM, et al. Monitorización de la oxigenación en pacientes críticos. Archivo Médico de Camagüey, 2011; 15: 697-704.

19. OLIVIERI PP, et al. Echo is a good, not perfect, measure of cardiac output in critically ill surgical patients. Journal Trauma Acute Care Surg, 2019; 87(2): 379-385

20. MERCADAL MJ, et al. Ecocardiografía funcional en la unidad de reanimación como monitor hemodinámico. Med Crit, 2017; 31(2): 84-92.

21. KOSTER G, VAN DER HORST ICC, Critical care ultrasonography as complementary variable in the diagnosis and management of circulatory shock. Current Opinion Critical Care, 2017; 23(4): 326-333. 